\title{
Acknowledgement to Reviewers of Journal of Intelligence in 2018
}

\author{
J. Intell. Editorial Office \\ MDPI, St. Alban-Anlage 66, 4052 Basel, Switzerland; jintelligence@mdpi.com \\ Published: 8 January 2019
}

\begin{abstract}
Rigorous peer-review is the corner-stone of high-quality academic publishing. The editorial team greatly appreciates the reviewers who contributed their knowledge and expertise to the journal's editorial process over the past 12 months. In 2018, a total of 48 papers were published in the journal, with a median time to first decision of 29 days and a median time to publication of 69 days. The editors would like to express their sincere gratitude to the following reviewers for their cooperation and dedication in 2018:
\end{abstract}

\begin{tabular}{|c|c|c|}
\hline Abele, Stephan & De Boeck, Paul & Hülür, Gizem \\
\hline Ahmetoglu, Gorkan & Demetriou, Andreas & Iorga, Magdalena \\
\hline Ambrose, Don & Detterman, Douglas & Jain, Pinky \\
\hline Anderson, Mike & Diaz-Sarachaga, Jose & Jayawickreme, Eranda \\
\hline Aschenbrenner, Andrew & Manuel & Jonason, Peter K. \\
\hline Atit, Kinnari & Dijk, Marijn Van & Kalalahti, Mira \\
\hline Ausloos, Marcel & Dresp-Langley, Birgitta & Kanaya, Tomoe \\
\hline Badger, Julia & Dunkel, Curtis & Karwowski, Maciej \\
\hline Baron, Jonathan & Fasko, Daniel & Kaufman, James \\
\hline Beauducel, Andre & Fayn, Kirill & Khan, Atif Ali \\
\hline Beaujean, Alexander & Fernandez, Sébastien & Kievit, Rogier \\
\hline Bergold, Sebastian & Figueredo, Aurelio José & Klimes-Dougan, Bonnie \\
\hline Boom, Jan & Fontaine, Johnny & Klose, Diana \\
\hline Bratko, Denis & Forgeard, Marie & Ko, Celine \\
\hline Broniatowski, David Andre & Frey, Meredith & Kornhaber, Mindy \\
\hline Brouwer, Rachel & Gardner, Howard & Kovacs, Kristof \\
\hline Cabeza De Baca, Tommy & Goethals, George & Kretzschmar, Andre \\
\hline Caemmerer, Jacqueline M. & Goldhammer, Frank & Kretzschmar, André \\
\hline Campbell, Frances A. & Golle, Jessika & Kyllonen, Patrick \\
\hline Chuderski, Adam & González Valero, Gabriel & Lakin, Joni M. \\
\hline Colom, Roberto & Graesser, Arthur & Laming, Donald Richard \\
\hline Conway, Andrew & Grossmann, Igor & John \\
\hline Corbin, Jonathan Charles & Hancock, Peter J.B. & Lapalme, Matthew \\
\hline Costa, Paul & Hein, Sascha D & Lautrey, Jacques \\
\hline Costello, Shane & Herzberg, Philipp Y. & Lechner, Clemens \\
\hline Coyle, Thomas R. & Hilbert, Sven & Lemaire, Patrick \\
\hline Dauvier, Bruno & Holt, Daniel & Leon Rubio, Jose Maria \\
\hline
\end{tabular}




Lesiuk, Teresa
Loi, Natasha
Luis Ubago, Jose
Marx, Peter
Marzocchi, Gian Marco
Marzoli, Daniele
Matta, Michael
Mattison, Richard
Mayer, Axel
McAbee, Samuel
Meara, Paul
Michalkiewicz, Martha
Mottus, Rene
Murphy, Kevin
Murphy, Raegan
Naglieri, Jack A.
Narvaez, Darcia
Nauta, Noks
Nęcka, Edward
Neubauer, Andreas
Núñez Pérez, José Carlos
Nußbeck, Fridtjof
Oshio, Atsushi
Panno, Angelo

Plucker, Jonathan

Randall, Jason

Ranger, Jochen

Rauthmann, John

Redifer, Jenni

Rey-Mermet, Alodie

Rinaldi, Luca

Rindermann, Heiner

Ruiz-Barquín, Roberto

Sajjadi, Pejman

Schmidt, Frank

Schmiedek, Florian

Schmitz, Florian

Schneider, W. Joel

Schroeders, Ulrich

Schubert, Anna-Lena

Schweizer, Karl

Shelton, Nicole

Simonton, Dean Keith

St Clair-Thompson, Helen

Stanovich, Keith

Staudinger, Ursula M.

Sternberg, Robert J.

Storek, Josephine
Stough, Lon

Svob Strac, Dubravka

Te Nijenhuis, Jan

Toplak, Maggie

Uttal, David

Van Der Linden, Dimitri

Van Der Maas, Han L.J.

Von Stumm, Sophie

Wacker, Jan

Wai, Jonathan

Wakeman, Shawnee

Waterhouse, Lynn

Watkins, Marley W.

Webb, Rose Mary

Weldon, Rebecca

Wenos, Jeanne

Weststrate, Nic

Wood, Dustin

Woodley, Michael

Zelazo, Philip David

Ziegler, Matthias

Zou, Liye

$\mathrm{Zu}$, Jiyun

(C) 2019 by the author. Licensee MDPI, Basel, Switzerland. This article is an open access article distributed under the terms and conditions of the Creative Commons Attribution (CC BY) license (http://creativecommons.org/licenses/by/4.0/). 